\title{
Evaporation residue cross-section measurements for ${ }^{16} \mathrm{O}+{ }^{203,205} \mathrm{Tl}$
}

\author{
J. Gehlot,,${ }^{1,2, *}$ A. M. Vinodkumar, ${ }^{2}$ N. Madhavan, ${ }^{1}$ S. Nath, ${ }^{1}$ A. Jhingan, ${ }^{1}$ T. Varughese,${ }^{1}$ Tathagata Banerjee,${ }^{1, \dagger}$ A. Shamlath, \\ P. V. Laveen, ${ }^{3}$ M. Shareef, ${ }^{3}$ P. Jisha,${ }^{2}$ P. Sandya Devi, ${ }^{4}$ G. Naga Jyothi, ${ }^{4}$ M. M. Hosamani, ${ }^{5}$ I. Mazumdar, ${ }^{6}$ V. I. Chepigin, ${ }^{7}$ \\ M. L. Chelnokov, ${ }^{7}$ A. V. Yeremin, ${ }^{7}$ A. K. Sinha, ${ }^{8}$ and B. R. S. Babu ${ }^{2}$ \\ ${ }^{1}$ Nuclear Physics Group, Inter University Accelerator Centre, Aruna Asaf Ali Marg, New Delhi 110067, India \\ ${ }^{2}$ Department of Physics, University of Calicut, Calicut 673635, India \\ ${ }^{3}$ Department of Physics, School of Mathematical and Physical Sciences, Central University of Kerala, Kasaragod 671314, India \\ ${ }^{4}$ Department of Nuclear Physics, Andhra University, Visakhapatnam 530003, India \\ ${ }^{5}$ Department of Studies in Physics, Karnatak University, Dharwad-580003, India \\ ${ }^{6}$ Tata Institute of Fundamental Research, Mumbai 400005, India \\ ${ }^{7}$ FLNR, JINR, Dubna 141980, Russia \\ ${ }^{8}$ UGC-DAE CSR, Indore 452017, India
}

(Received 10 August 2018; revised manuscript received 15 December 2018; published 22 March 2019)

\begin{abstract}
Evaporation residue cross sections for the ${ }^{16} \mathrm{O}+{ }^{203,205} \mathrm{Tl}$ reactions were measured at laboratory beam energies in the range of 82-113 MeV using a gas-filled separator. Transmission efficiency of the separator was estimated using a calibration reaction ${ }^{16} \mathrm{O}+{ }^{197} \mathrm{Au}$ and by simulating the evaporation residues angular distributions. Statistical model calculations were performed for both the measured systems. These calculations overestimate the experimental evaporation residue cross sections. This could be attributed to the presence of noncompound nuclear fission. An estimation of noncompound nuclear fission contribution was carried out. Comparison with neighboring systems shows that a slight change in the entrance channel or the compound nucleus properties makes a large difference in evaporation residue cross sections.
\end{abstract}

DOI: 10.1103/PhysRevC.99.034615

\section{INTRODUCTION}

Heavy-ion fusion-fission reaction dynamics has been an active field of study for the past several decades and it is still not fully understood, especially for the heavier nuclei $(A \geqslant 200 \mathrm{amu})$ around the barrier. Heavy-ion fusion reactions are particularly important as they are the most successful mechanisms for superheavy element (SHE) production [1-3].

One-dimensional barrier penetration model explains the nuclear fusion quite reasonably. However, it underestimates the fusion cross sections near and below the Coulomb barrier. The enhancement of experimental reaction cross section was found to be due to the coupling of various internal degrees of freedom, such as static deformation of the collision partners, collective surface vibrations, and transfer channels, etc., with the relative motion $[4,5]$. The tunneling through the barrier and subsequent capture are enhanced by the coupling of these internal degrees of freedom around the barrier.

As per the compound nucleus $(\mathrm{CN})$ hypothesis, the dinuclear system follows a long dynamical path during which it equilibrates in all degrees of freedom or reseparates into fission-like fragments. The $\mathrm{CN}$ which de-excites via particle evaporation or $\gamma$ emission and survives fission, ends up as various evaporation residues (ER). Formation of these ERs

\footnotetext{
*jagdishgehlot@iuac.res.in; jagdishgehlot@gmail.com

†Present address: Department of Nuclear Physics, Australian National University, Canberra, ACT 2601, Australia.
}

depends on the capture probability, $\mathrm{CN}$ formation probability, and its survival probability against fission. The ER cross section is given as

$$
\sigma_{\mathrm{ER}}=\sigma_{\text {cap }} \times P_{\mathrm{CN}} \times P_{\text {surv }},
$$

where $\sigma_{\text {cap }}$ is capture cross section, $P_{\mathrm{CN}}$ is $\mathrm{CN}$ formation probability, and $P_{\text {surv }}$ is its survival probability against fission. For light and very asymmetric systems, merely overcoming the capture barrier is sufficient for $\mathrm{CN}$ formation but this is not the case for heavier systems, as they may reseparate before $\mathrm{CN}$ formation.

It was observed that at higher excitation energies, the prescission neutron, charge particle, and giant dipole resonance (GDR) $\gamma$-decay multiplicities exceed the statistical model predictions [6]. Inclusion of dissipation or viscosity effects into the Bohr-Wheeler formalism [7] is required to explain these higher multiplicities. These effects reduce the fission and increase the particle and $\gamma$ emission in the presaddle region and thereby the ER cross section. However, often the ER cross sections cannot be reproduced with the same strength of dissipation $[8,9]$. Usually a smaller dissipation strength is required to reproduce the ER data in comparison with that required for prescission multiplicity data. For a number of systems, the fission is enhanced by reducing the height of the liquid drop model (LDM) fission barrier to fit the ER cross sections [10,11].

While explaining the reaction excitation function, any deviation from the standard statistical model predictions is 
generally attributed to either the presence of proton or neutron shell closure in the compound nucleus or to the contribution from noncompound nuclear fission (NCNF). While shell effects are known to give extra stability against fission, NCNF reduces the probability of complete fusion. Their influences on fusion-fission dynamics have been studied extensively.

Around the $A=200$ amu mass region and beyond, $\mathrm{CN}$ fission and NCNF coexist and it is very difficult to experimentally disentangle these processes, as they have overlapping experimental signatures [12]. Recently, a systematic analysis of existing ER cross-section data in the mass region of 170-220 amu [13] identified the approximate boundaries from where the average fusion probability $\left\langle\mathrm{P}_{\mathrm{CN}}\right\rangle$ deviates from unity.

Effects of proton and neutron shell closures $(Z=82$ and/or $N=126$ ) on survival of ER against fission [14-18], fission fragment angular anisotropy $[19,20]$, mass distribution [21], quassifission [22], and other observables like $\alpha$ decay [23] have been studied. The ERs, being the true signature of $\mathrm{CN}$ formation, can be used to explore the onset of NCNF and also the dissipative and shell effects. Though extensive work is reported on ER measurements by various groups, there are still several unanswered questions requiring further studies to get a complete picture of the nuclear reaction dynamics leading to a better understanding of SHE formation.

In the present work, we report the measurements of ER cross sections for ${ }^{16} \mathrm{O}+{ }^{203,205} \mathrm{Tl}$ reactions. To the best of our knowledge there are no reported measurements on these reactions to date. The experimental details are given in Sec. II, data analysis is given in Sec. III followed by results and discussion in Sec. IV, and, finally, a brief summary in Sec. V.

\section{EXPERIMENTAL DETAILS}

Measurements were carried out at the 15 UD Pelletron accelerator facility of IUAC, New Delhi (India) [24,25]. A pulsed beam of ${ }^{16} \mathrm{O}$, with pulse separation of $2 \mu \mathrm{s}$ and a pulse width of $1 \mathrm{~ns}$ was used to bombard ${ }^{203} \mathrm{Tl}$ and ${ }^{205} \mathrm{Tl}$ targets of thicknesses $\sim 175 \pm 10 \mu \mathrm{g} / \mathrm{cm}^{2}$ and $155 \pm 10 \mu \mathrm{g} / \mathrm{cm}^{2}$, respectively, with thin $\left(\sim 35 \mu \mathrm{g} / \mathrm{cm}^{2}\right)$ carbon backing [26]. Experiment was carried out at laboratory projectile energies $\left(E_{\text {lab }}\right)$ in the range of $82-113 \mathrm{MeV}$.

The ERs were separated from the background events using the first stage of the HYbrid Recoil mass Analyzer (HYRA) [27] operated in gas-filled mode. HYRA is a dual-mode, dualstage spectrometer/separator with its first stage capable of operating in gas-filled or vacuum mode in normal kinematics and both stages in vacuum mode in inverse kinematics. The magnetic field region was filled with helium gas at a pressure of 0.15 Torr. The gas-filled region was separated from the beam line vacuum using a diamond-like carbon foil of $\sim 3 \mu \mathrm{m}$ thickness and $15 \mathrm{~mm}$ diameter.

In the target chamber, two silicon detectors were placed at a laboratory angle of $25^{\circ}$, with respect to beam direction, in the horizontal plane, for absolute normalization of ER cross sections. At the focal plane (FP) of HYRA, a multiwire proportional counter (MWPC) [28], having an active area of $15.0 \times 5.0 \mathrm{~cm}^{2}$, followed by a double-sided silicon strip detector were used to record the ERs. A very thin $(0.5 \mu \mathrm{m})$ mylar foil was used as the entrance window of the MWPC in order to minimize the ER energy loss prior to its detection in the MWPC. The effective active area of the MWPC was reduced to $11.0 \times 5.0 \mathrm{~cm}^{2}$, due to the foil supporting flange. The MWPC was operated at $\sim 3$ mbar of isobutane. Magnetic fields were optimized around the calculated values [29], at each energy point, by maximizing the ER yield at the focal plane. Times of flight (TOFs) of the ERs were recorded taking MWPC anode signal as "START" and RF signal of beam pulsing system as "STOP." Yields of ERs were extracted from the coincidence spectrum between energy loss $\Delta E$ (from the cathode of MWPC) and TOF.

\section{DATA ANALYSIS}

\section{A. ER cross section}

The number of events selected from a two-dimensional spectrum of $\Delta E$ vs. TOF has been used to obtain the ER cross section using the equation

$$
\sigma_{\mathrm{ER}}=\frac{Y_{\mathrm{ER}}}{Y_{\mathrm{mon}}}\left(\frac{d \sigma}{d \Omega}\right)_{\text {Ruth }} \Omega_{\mathrm{mon}} \frac{1}{\varepsilon},
$$

where $\sigma_{\mathrm{ER}}$ is the ER cross section (in mb), $\mathrm{Y}_{\mathrm{ER}}$ is ER yield at the focal plane of HYRA, $Y_{\text {mon }}$ is the average yield in monitor detectors, $\varepsilon$ is the transmission efficiency of the separator, and $\Omega_{\text {mon }}$ is the solid angle (in Sr) subtended by each monitor detector. $\left(\frac{d \sigma}{d \Omega}\right)_{\text {Ruth }}$ is the differential Rutherford scattering cross section (in $\mathrm{mb} / \mathrm{Sr}$ ) in the laboratory system at chosen scattering angle and energy.

\section{B. Transmission efficiency}

In Eq. (2), apart from the experimental observables, transmission efficiency $(\varepsilon)$ of the separator is an important factor, which needs to be known precisely in order to extract absolute ER cross sections. Transmission efficiency of the separator is the ratio of the ERs detected at the focal plane of the separator to the total number of ERs produced at target site and depends on several factors, such as entrance channel mass asymmetry, beam energy, geometrical acceptance of the separator, target thickness (due to multiple scattering), helium gas pressure in the separator, focal plane detector size, and presence of some short-lived isomers [30]. Isomers with a half-life less than ER TOF may result in Auger processes and thereby change the ER charge state in flight and may deviate its path drastically and hence affect the transmission (for vacuum mode separators). For gas-filled separators the dilute gas (He in this case) is expected to reset the charge state along the trajectory. Moreover, in gas-filled separators, the ERs with different charge states tend toward a mean charge state through multiple collisions in the dilute gas medium and, on average, follow a mean trajectory. Hence, the presence of short-lived isomers is not expected to affect the transmission drastically for gas-filled separators.

The transmission efficiency is generally measured during the experiment, using a $\gamma$ detector at target site, via characteristic $\gamma$ detection in both coincidence and singles mode [31]. Such measurements involve coincidence studies between 
TABLE I. HYRA transmission efficiency values for the calibration reaction ${ }^{16} \mathrm{O}+{ }^{197} \mathrm{Au}$ at different beam energies using Brinkmann et al.'s [33] ER data.

\begin{tabular}{lccc}
\hline \hline Reaction & $E_{\text {lab }}(\mathrm{MeV})$ & $\sigma_{\mathrm{ER}}(\mathrm{mb})$ & $\varepsilon_{\mathrm{HYRA}}(\%)$ \\
\hline${ }^{16} \mathrm{O}+{ }^{197} \mathrm{Au}$ & 93.4 & $168.3 \pm 16.8$ & $0.13 \pm 0.03$ \\
& 99.5 & $131.1 \pm 13.1$ & $0.15 \pm 0.03$ \\
& 105.6 & $98.9 \pm 9.9$ & $0.16 \pm 0.03$ \\
& 113.8 & $67.8 \pm 6.8$ & $0.16 \pm 0.03$ \\
\hline \hline
\end{tabular}

characteristic $\gamma$ rays at target site and ERs at focal plane. These measurements require longer beam time. In addition to this, we should keep in mind that the background at target site will also be a serious problem in accurate estimation of transmission efficiency. Therefore, at times, it may be necessary to simulate it or scale from a nearby similar reaction. In the present investigation, we have adopted the method of scaling using a nearby system [32]. A calibration reaction forming ${ }^{213} \mathrm{Fr}$ through ${ }^{16} \mathrm{O}+{ }^{197} \mathrm{Au}$ was used to estimate the transmission efficiency. For this, we have used the ER cross-section data from Brinkmann et al. [33]. The estimated transmission efficiencies for ${ }^{16} \mathrm{O}+{ }^{197} \mathrm{Au}$ are given in Table I.

For scaling the efficiency to our measured reactions, we simulated ER angular distributions for the calibration reaction ${ }^{16} \mathrm{O}+{ }^{197} \mathrm{Au}$ and for presently measured reactions, viz. ${ }^{16} \mathrm{O}+{ }^{203,205} \mathrm{Tl}$ using the code PACE4 [34,35]. Transmission efficiencies for the present systems were then scaled from the calibration reaction by comparing the fraction of their respective angular distributions passing through the geometrical acceptance of HYRA, i.e., $9.5^{\circ}$, in a way similar to our previous measurements [32,36-41]. Figure 1 shows the angular distributions of ERs at different beam energies for the measured reactions, viz. ${ }^{16} \mathrm{O}+{ }^{203,205} \mathrm{Tl}$, along with those for the calibration reaction, i.e., ${ }^{16} \mathrm{O}+{ }^{197} \mathrm{Au}$. The variation of angular distributions are in accordance with the kinematics. With increasing energy, the angular distribution should be forward focused. The calibration reaction ${ }^{16} \mathrm{O}+{ }^{197} \mathrm{Au}$ has more fraction of $\alpha$-evaporation channels than non- $\alpha$ evaporation channels, hence having broader distribution. At higher energies, ${ }^{16} \mathrm{O}+{ }^{203,205} \mathrm{Tl}$ systems also have more $\alpha$ evaporation channels and the shape of ER angular distribution is quite similar to that for ${ }^{16} \mathrm{O}+{ }^{197} \mathrm{Au}$ reaction. Transmission efficiencies thus obtained for the reactions ${ }^{16} \mathrm{O}+{ }^{203} \mathrm{Tl}$ and ${ }^{16} \mathrm{O}+{ }^{205} \mathrm{Tl}$ are listed in column 5 of Table II.

\section{C. $\alpha$-decay effects on ER transmission efficiency}

If the half-life of the $\alpha$-decaying ER is less than its TOF, then it may decay inside the separator and the push given by the emitted $\alpha$ particle may deflect the heavy recoil from its path and thus reduce its transmission through the separator. The $\alpha$-decaying ERs produced in these reactions are mostly actinium, radium, and francium isotopes. Statistical model code HIVAP [42,43] was used to check the major decay channels and their relative population in the studied reactions in the measured energy range. Figure 2 shows the individual ER yields for ${ }^{16} \mathrm{O}+{ }^{203} \mathrm{Tl}$ and ${ }^{16} \mathrm{O}+{ }^{205} \mathrm{Tl}$ reactions using HIVAP $[42,43]$ at different beam energies. Only ER channels with relative yield of more than $1 \%$ of the total ER were considered.

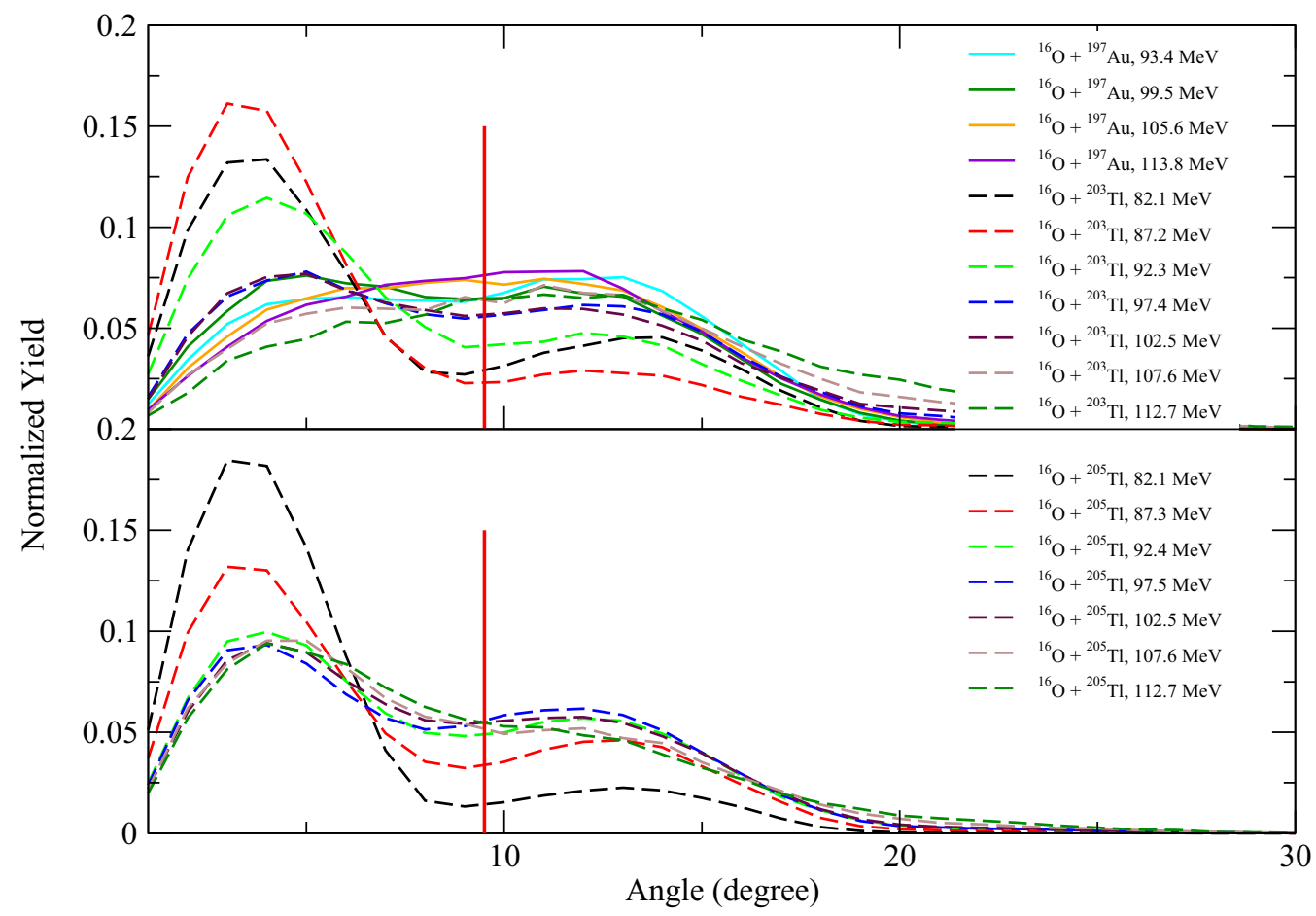

FIG. 1. Angular distributions of ERs at different beam energies using PACE4 [34,35]. The vertical line at $9.5^{\circ}$ denotes the HYRA angular acceptance. 
TABLE II. HYRA transmission efficiency $\epsilon_{\mathrm{HYRA}}$ for ${ }^{16} \mathrm{O}+{ }^{203} \mathrm{Tl}$ and ${ }^{16} \mathrm{O}+{ }^{205} \mathrm{Tl}$ reactions scaled from calibration reaction ${ }^{16} \mathrm{O}+{ }^{197} \mathrm{Au}$, fraction of ERs reaching FP surviving the in-flight $\alpha$ decay $\left(\eta_{\alpha \text {-decay }}\right)$, and respective ER cross section $\left(\sigma_{\mathrm{ER}}\right)$ at different energies.

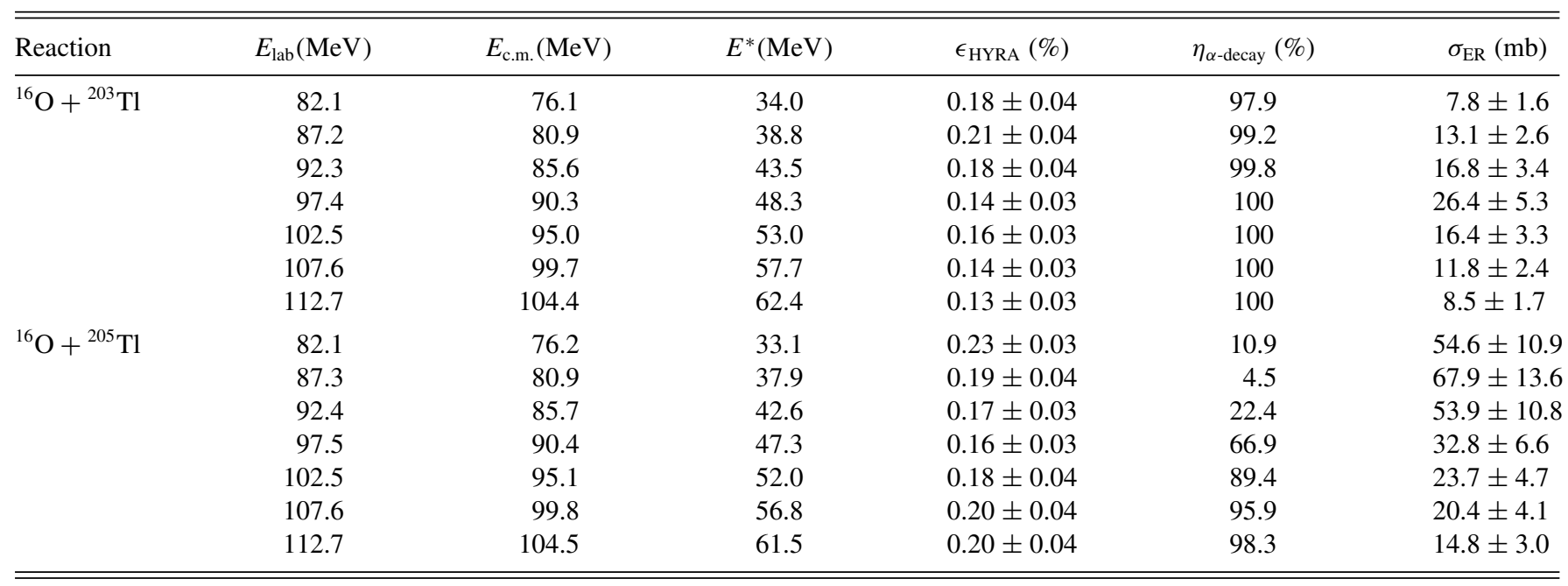

For the ${ }^{16} \mathrm{O}+{ }^{203} \mathrm{Tl}$ case, most of the ERs, as predicted by HIVAP, have sufficiently long half-lives to reach focal plane with hardly any loss due to decay. However, for the reaction ${ }^{16} \mathrm{O}+{ }^{205} \mathrm{Tl}$, at lower energies, the exit channels $3 n, 4 n$, or $\alpha 2 n$, produce a significant number of the ERs ${ }^{218} \mathrm{Ac},{ }^{217} \mathrm{Ac}$, and ${ }^{215} \mathrm{Fr}$, respectively, having half-lives of $1.08 \mu \mathrm{s}, 69 \mathrm{~ns}$, and $86 \mathrm{~ns}$, respectively [44], much less than the average TOF through the gas-filled separator HYRA. These nuclei decay during the flight as per the radioactive decay law. As a result, they are not transmitted fully through the separator.
The $\alpha$ particles emitted in extreme forward or backward direction will not deflect the ERs from their path. For an ER traveling along the central trajectory, the push given by emitted $\alpha$, with an energy for the present case $(\sim 9 \mathrm{MeV})$, is sufficient to deflect the ER away from the view of the focal plane detector. Only ERs decaying at the very end of the flight path manage to be detected in the focal plane detector. Therefore, as an extreme approximation, it is assumed that any ER decaying in-flight will not reach the focal plane detector. The transmission loss due to in-flight decay of short-lived ERs is compensated considering their relative population,

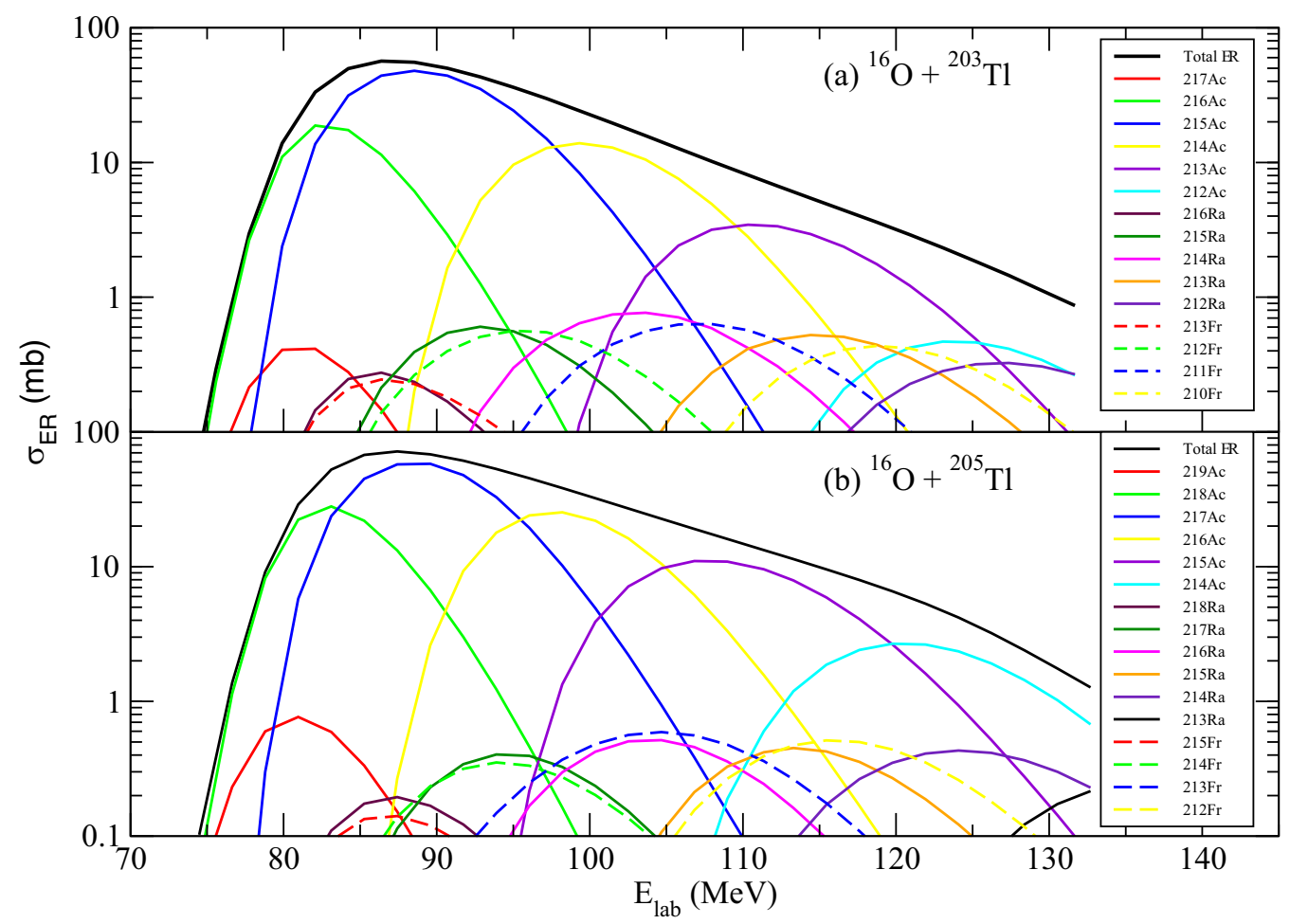

FIG. 2. Yield of individual exit channels for (a) ${ }^{16} \mathrm{O}+{ }^{203} \mathrm{Tl}$ and (b) ${ }^{16} \mathrm{O}+{ }^{205} \mathrm{Tl}$ reactions at different beam energies by HIVAP [42,43]. 
respective half-life, and the TOF through the separator. Column 6 of Table II shows the fraction of ERs entering the separator, which survives the in-flight decay and reaches the FP. The efficiency values listed in column 5 are further multiplied by these fractions to have absolute efficiencies. The ER cross sections thus obtained from the experiment are summarized in column 7 of Table II. The overall errors in the obtained cross sections are less than $20 \%$.

\section{Statistical model calculations}

Experimental ER cross sections were analyzed using HIVAP code [42,43], which incorporates a potential-barrier passing model with standard statistical model (SSM). We have used the standard parameter set suggested by ReisdorfSchadel [43]. ER cross sections calculated for strongly fissile $\mathrm{CN}$ at energies well above the fusion barrier [45], are relatively insensitive to the form of the nuclear potential [46,47], and are mainly determined by SSM parameters which describe the deexcitation of $\mathrm{CN}$. The macroscopic parameters of the nuclear level densities in fission and evaporation channels are provided by ratios of level densities, $\widetilde{a}_{f} / \widetilde{a}_{n} \geqslant 1$, due to different shapes at the saddle and equilibrium states [42,43]. $\tilde{a}_{f}$ is the level density parameter at fission saddle point and $\widetilde{a}_{n}$ is after neutron evaporation. A damping constant of $18.5 \mathrm{MeV}$ $[42,43,48]$ was used to take care of ground-state shell effects which have been neglected at the saddle point. Empirical masses [49] were used to calculate ground-state shell corrections, excitation, and separation energies. As shown by
Sagaidak et al. [10], calculations performed in this work, at above fusion barrier energies, depend only on one adjustable scaling parameter $k_{f}$ of the LD fission barrier, i.e., the fission barrier is given by $B_{f}(\ell)=k_{f} B_{f}^{\mathrm{LD}}(\ell)-\delta W_{\text {g.s. }}$, where $B_{f}^{\mathrm{LD}}(\ell)$ is the rotating LD fission barrier and $\delta W_{\text {g.s. }}$ is the difference between the empirical and LD masses.

\section{RESULTS AND DISCUSSION}

In Fig. 4 and Fig. 5, we plot ER excitation functions for ${ }^{16} \mathrm{O}+{ }^{205} \mathrm{Tl}$ and ${ }^{16} \mathrm{O}+{ }^{203} \mathrm{Tl}$, respectively. Statistical model calculations, using HIVAP $[42,43]\left(k_{f}=1\right)$, overpredict the experimental ER cross sections, which might be due to the presence of NCNF in these reactions. A reduced fission barrier $\left(k_{f}<1\right)$ may be required to reproduce the experimental ER cross sections. However, this alone does not confirm the presence of NCNF. The reduced fission barrier is rather a measure of the product $P_{\mathrm{CN}} \times P_{\text {surv }}$ being less than unity.

NCNF is usually not expected in reactions induced by ${ }^{16} \mathrm{O}$ and projectiles lighter than it. However, a recent systematic study by Banerjee et al. [13] hints that NCNF contribution may exist even in ${ }^{16} \mathrm{O}$-induced reactions. They have shown the variation of $\left\langle\mathrm{P}_{\mathrm{CN}}\right\rangle$ with entrance channel mass asymmetry $\eta$, charge product $Z_{p} Z_{t}$, and $\mathrm{CN}$ fissility $\chi_{\mathrm{CN}}$. None of these parameters were found adequate to be used as a single scaling variable to determine $\left\langle\mathrm{P}_{\mathrm{CN}}\right\rangle$. The authors identified the approximate boundaries where $\left\langle\mathrm{P}_{\mathrm{CN}}\right\rangle$ starts deviating from unity. The presently measured reactions ${ }^{16} \mathrm{O}+{ }^{203} \mathrm{Tl}$ and ${ }^{16} \mathrm{O}+{ }^{205} \mathrm{Tl}$, with $Z_{p} Z_{t}=648, \chi_{\mathrm{CN}}=0.758,0.755$ and

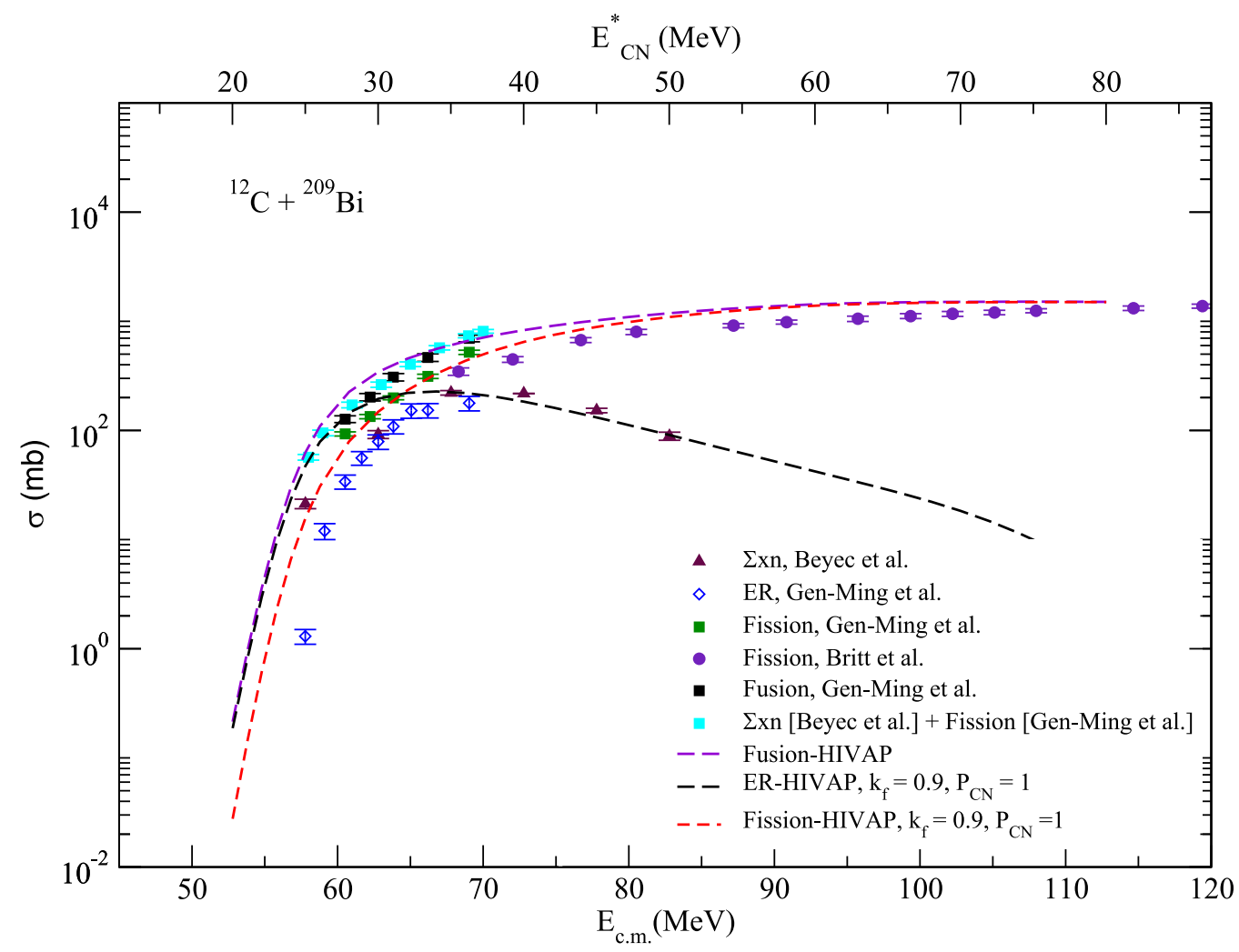

FIG. 3. Experimental ER and fission cross sections for the ${ }^{12} \mathrm{C}+{ }^{209} \mathrm{Bi}$ reaction (Beyec et al. [14], Gen-Ming et al. [51], Britt et al. [52]) along with HIVAP calculations. 


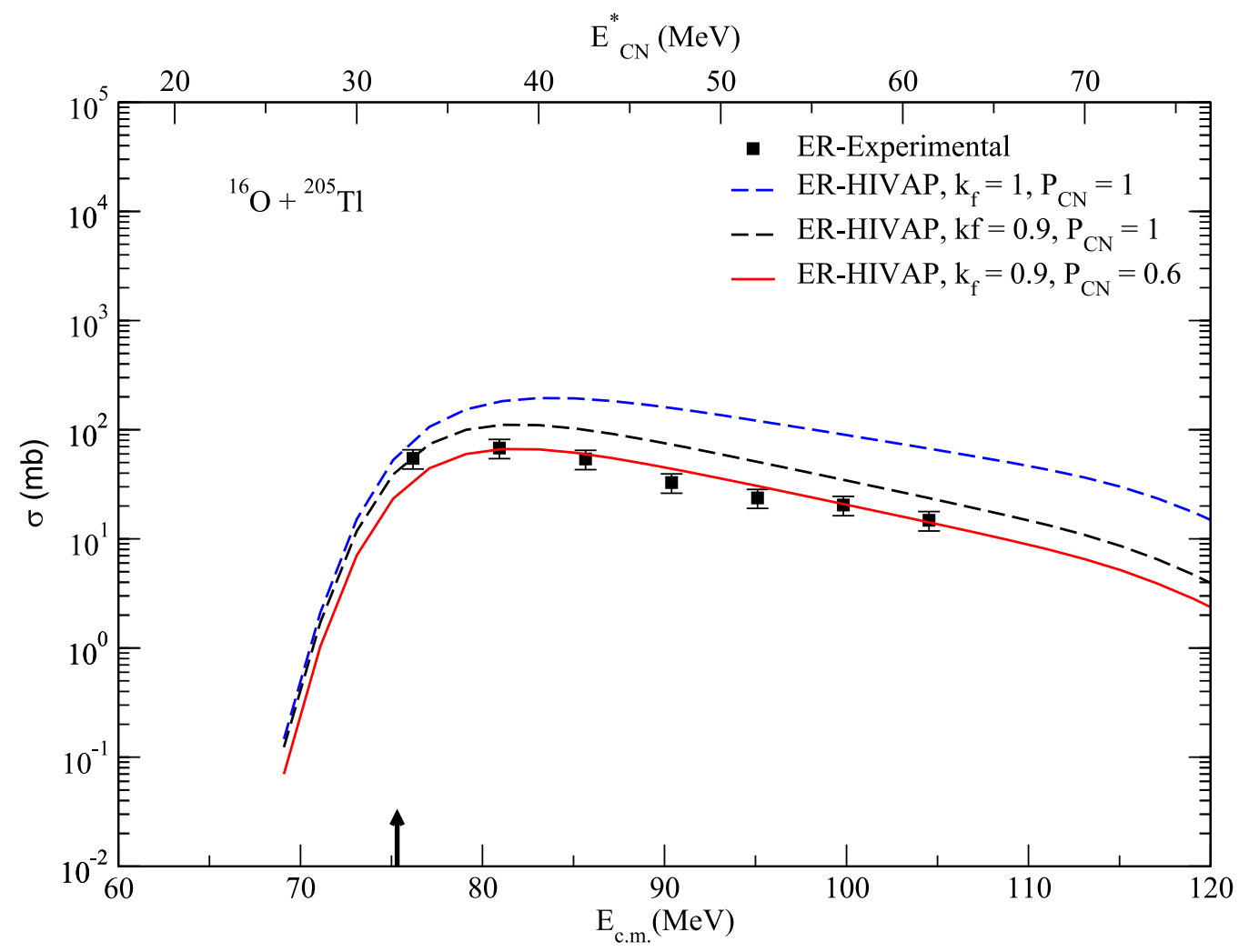

FIG. 4. Experimental ER cross sections for ${ }^{16} \mathrm{O}+{ }^{205} \mathrm{Tl}$ reaction along with HIVAP calculations.

$\eta=0.854$ and 0.855 , respectively, lie beyond these boundaries, indicating a probable contribution from NCNF. Here $\eta$ is given by $\eta=\frac{\left|A_{p}-A_{t}\right|}{A_{p}+A_{t}}, \mathrm{~A}_{p}$ and $\mathrm{A}_{t}$ being mass numbers of projectile and target, respectively. $\chi_{\mathrm{CN}}$ is calculated as given in Ref. [50].

In order to quantify the contribution from NCNF, we followed a method similar to the one prescribed by Sagaidak et al. [10]. The two reactions ${ }^{12} \mathrm{C}+{ }^{209} \mathrm{Bi}[14,51,52]$ and ${ }^{16} \mathrm{O}+{ }^{205} \mathrm{Tl}$ produce the same $\mathrm{CN},{ }^{221} \mathrm{Ac}$. The fission barrier scaling factor $k_{f}$ is fixed using the asymmetric reaction ${ }^{12} \mathrm{C}+{ }^{209} \mathrm{Bi}$, where NCNF is least expected. The experimental cross sections are in considerable agreement with HIVAP calculations with $k_{f}=0.9, P_{\mathrm{CN}}=1$ (Fig. 3). In most of these measurements [51,52], projectile energy was reduced using thick degrader foils, thereby causing large uncertainties in projectile energy at lower energies.

We calculated the ER cross section for ${ }^{16} \mathrm{O}+{ }^{205} \mathrm{Tl}$ with the same fission barrier scaling $\left(k_{f}=0.9\right.$, similar to ${ }^{12} \mathrm{C}+$ ${ }^{209} \mathrm{Bi}$ reaction). However $P_{\mathrm{CN}}=1$ is not able to explain the observed ER cross section. A value $P_{\mathrm{CN}}=0.6$ is able to reproduce our results very well (Fig. 4). Since the ${ }^{16} \mathrm{O}+{ }^{203} \mathrm{Tl}$ and ${ }^{16} \mathrm{O}+{ }^{205} \mathrm{Tl}$ systems are very nearly identical, we took the same barrier scaling for ${ }^{16} \mathrm{O}+{ }^{203} \mathrm{Tl}$ also and extracted the similar value of $P_{\mathrm{CN}}$ (Fig. 5).

In order to see the change in $P_{\mathrm{CN}}$ due to change in $k_{f}$, we varied $k_{f}$ for the ${ }^{12} \mathrm{C}+{ }^{209} \mathrm{Bi}$ reaction (keeping the HIVAP predictions within the acceptable limits of the experimental cross sections) from 0.89 to 0.91 . Calculations for ${ }^{16} \mathrm{O}+{ }^{205} \mathrm{Tl}$ with $k_{f}=0.89$ give $P_{\mathrm{CN}} \sim 0.65$ and with $k_{f}=0.91, P_{\mathrm{CN}} \sim$
0.55. Accordingly, we have considered $P_{\mathrm{CN}}=0.6 \pm 0.05$ for the systems studied.

In both cases the $P_{\mathrm{CN}}$ so obtained is less than 1, implying there is a possible contribution from NCNF. Considering the scarcity of such measurements with lighter beams and the poor energy definition of the projectile $\left({ }^{12} \mathrm{C}\right)$, in the ${ }^{12} \mathrm{C}+{ }^{209} \mathrm{Bi}$ reaction $[14,51,52]$, the conclusion needs to be established by studying these reactions using other probes. Also more studies are required on few other systems to unambiguously establish the contribution from NCNF.

One can notice in Fig. 4 and Fig. 5 that the measured cross sections for ${ }^{16} \mathrm{O}+{ }^{205} \mathrm{Tl}$ agree with the HIVAP $\left(k_{f}=\right.$ $\left.0.9, P_{\mathrm{CN}}=0.6\right)$ predictions, for all energies studied while the measured values for ${ }^{16} \mathrm{O}+{ }^{203} \mathrm{Tl}$ are substantially less than the statistical model predictions near the Coulomb barrier energies. Also one can infer from Table II (column 7) that the experimental ER cross section for ${ }^{16} \mathrm{O}+{ }^{203} \mathrm{Tl}$ is significantly lower than that of ${ }^{16} \mathrm{O}+{ }^{205} \mathrm{Tl}$ near the Coulomb barrier energies. Though more studies are needed to understand this difference in this energy region, we find similar examples in the literature $[33,53,54]$. When we compare the ER cross sections of ${ }^{16} \mathrm{O}+{ }^{208} \mathrm{~Pb}[33,53]$ with that of ${ }^{16} \mathrm{O}+{ }^{204} \mathrm{~Pb}[54]$, we see a similar dip in the ${ }^{16} \mathrm{O}+{ }^{204} \mathrm{~Pb}$ case near the Coulomb barrier. The reduced ER cross sections for both these systems are shown in Fig. 6, and one could observe this similar deviation between ${ }^{16} \mathrm{O}+{ }^{204} \mathrm{~Pb}$ and ${ }^{16} \mathrm{O}+{ }^{208} \mathrm{~Pb}$.

We have made a comparison of the presently measured systems with the ${ }^{12} \mathrm{C},{ }^{16} \mathrm{O}$, and ${ }^{19} \mathrm{~F}$ induced reactions in the similar mass region. Comparison of reduced ER cross sections 


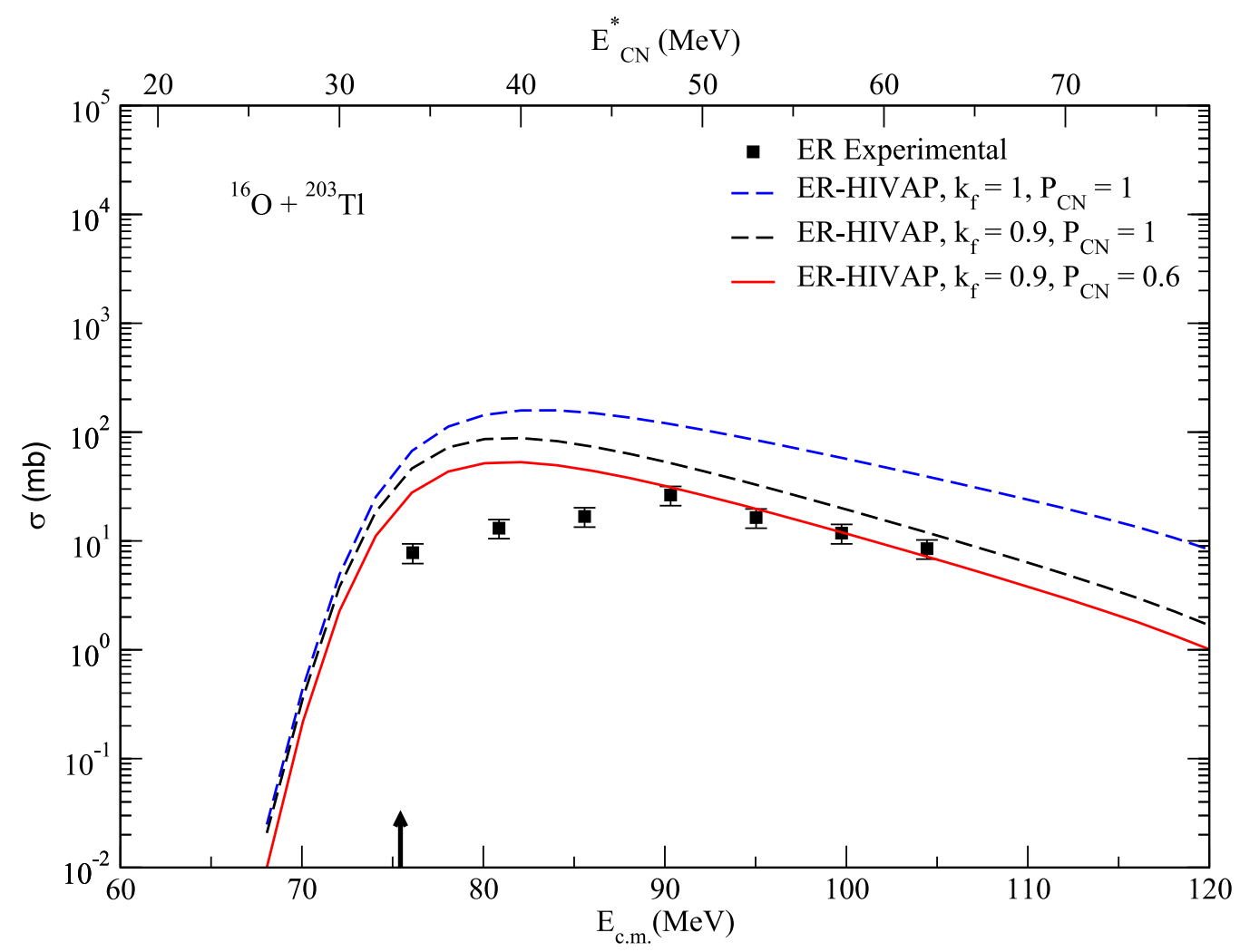

FIG. 5. Experimental ER cross sections for ${ }^{16} \mathrm{O}+{ }^{203} \mathrm{Tl}$ reaction along with HIVAP calculations.

$\left(\widetilde{\sigma}_{\mathrm{ER}}=\frac{\sigma_{\mathrm{ER}}}{\pi \lambda^{2}}\right)$ of the measured systems with the neighboring systems (Fig. 6) shows the effect of mass asymmetry in the entrance channel on $\sigma_{\mathrm{ER}}$ and a probable contribution from
NCNF. The comparison also shows that a slight change in the entrance channel or the $\mathrm{CN}$ properties makes a large difference in ER cross sections. More measurements are

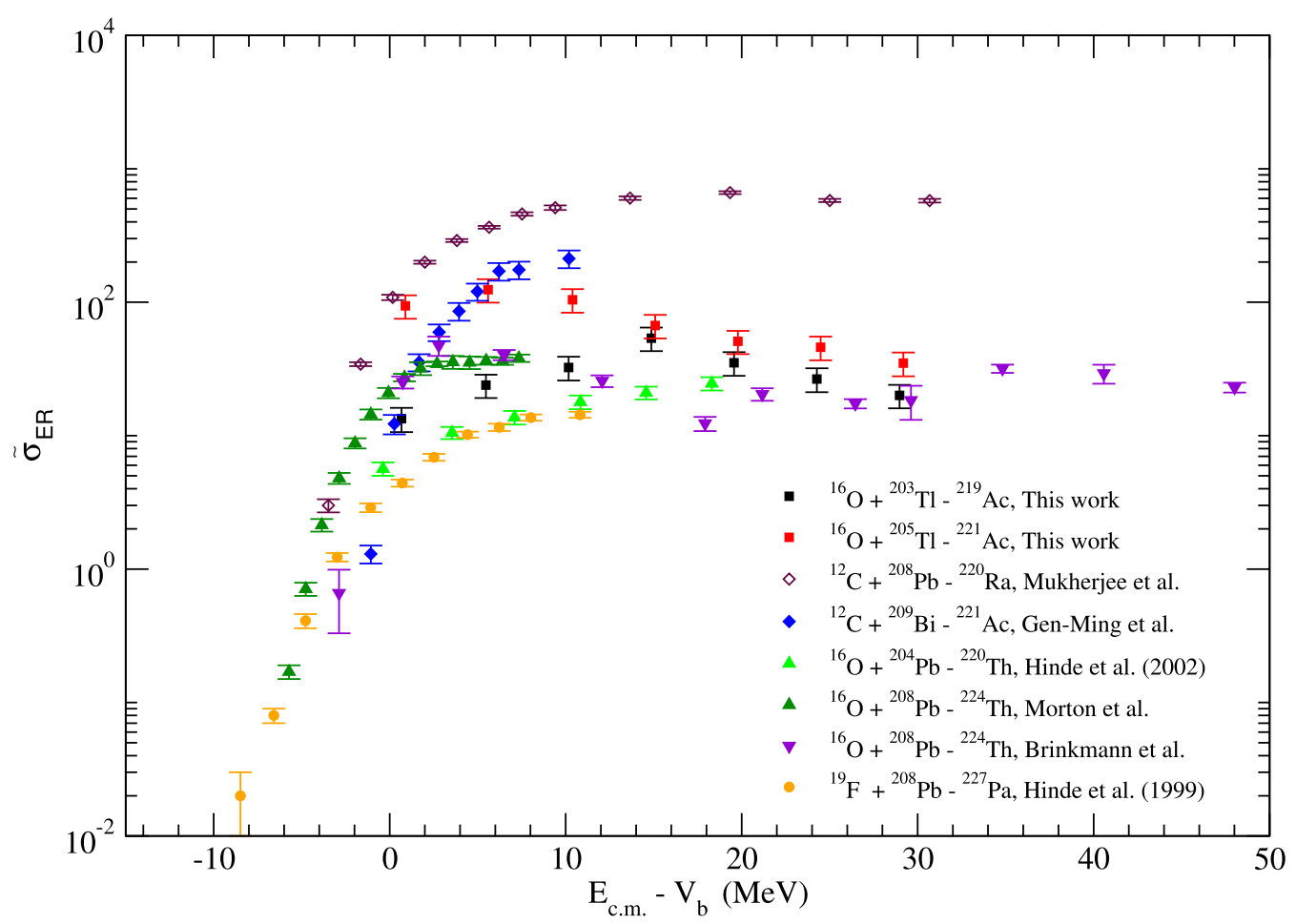

FIG. 6. Comparison of the reduced ER cross sections for ${ }^{16} \mathrm{O}+{ }^{203} \mathrm{Tl}$ and ${ }^{16} \mathrm{O}+{ }^{205} \mathrm{Tl}$ reactions with nearby systems (Brinkmann et al. [33], Gen-Ming et al. [51], Morton et al. [53], Hinde et al. (2002) [54], Hinde et al. (1999) [55], Mukherjee et al. [56]). 
needed to have a better understanding of nuclear reaction dynamics.

\section{SUMMARY}

Evaporation residue cross sections have been measured for the systems ${ }^{16} \mathrm{O}+{ }^{203} \mathrm{Tl}$ and ${ }^{16} \mathrm{O}+{ }^{205} \mathrm{Tl}$ near and well above the Coulomb barrier. There are no reported measurements on these systems and as such our results are the first measurements as far as we know. Statistical model calculations using HIVAP overestimates the evaporation residue cross sections. By introducing the fission barrier scaling factor $k_{f}=0.9$ and compound nucleus formation probability $P_{\mathrm{CN}}=0.6$, HIVAP calculations agree with the experimental values for both the reactions in the present study. This suggests that significant contribution from noncompound nuclear fission processes are probably responsible for the observed evaporation residue cross-section reduction. Comparison of reduced evaporation residue cross sections with neighboring systems shows that a slight change in the entrance channel or in the properties of compound nucleus makes a significant difference in the observed cross sections. More studies are further required to get a thorough picture of noncompound nuclear fission contribution and the physics thereof.

\section{ACKNOWLEDGMENTS}

We thank S. R. Abhilash for his help in isotopic target fabrication and Pelletron group of IUAC, New Delhi, for providing stable pulsed beam of required energy. We also thank E. Prasad Nair for important discussions during the course of this work.
[1] S. Hofmann and G. Münzenberg, Rev. Mod. Phys. 72, 733 (2000).

[2] J. H. Hamilton, S. Hofmann, and Y. T. Oganessian, Annu. Rev. Nucl. Part. Sci. 63, 383 (2013).

[3] Y. T. Oganessian and V. K. Utyonkov, Rep. Prog. Phys. 78, 036301 (2015).

[4] M. Dasgupta, D. J. Hinde, N. Rowley, and A. M. Stefanini, Annu. Rev. Nucl. Part. Sci. 48, 401 (1998).

[5] M. Beckerman, Rep. Prog. Phys. 51, 1047 (1988).

[6] M. Thoennessen and G. F. Bertsch, Phys. Rev. Lett. 71, 4303 (1993).

[7] N. Bohr and J. A. Wheeler, Phys. Rev. 56, 426 (1939).

[8] I. Dioszegi, N. P. Shaw, I. Mazumdar, A. Hatzikoutelis, and P. Paul, Phys. Rev. C 61, 024613 (2000).

[9] P. N. Nadtochy, E. G. Ryabov, A. E. Gegechkori, Yu. A. Anischenko, and G. D. Adeev, Phys. Rev. C 85, 064619 (2012).

[10] R. N. Sagaidak, G. N. Kniajeva, I. M. Itkis, M. G. Itkis, N. A. Kondratiev, E. M. Kozulin, I. V. Pokrovsky, A. I. Svirikhin, V. M. Voskressensky, A. V. Yeremin, L. Corradi, A. Gadea, A. Latina, A. M. Stefanini, S. Szilner, M. Trotta, A. M. Vinodkumar, S. Beghini, G. Montagnoli, F. Scarlassara, D. Ackermann, F. Hanappe, N. Rowley, and L. Stuttge, Phys. Rev. C 68, 014603 (2003).

[11] R. N. Sagaidak and A. N. Andreyev, Phys. Rev. C 79, 054613 (2009).

[12] D. J. Hinde, R. du Rietz, and M. Dasgupta, EPJ Web Conf. 17, 04001 (2011).

[13] T. Banerjee, S. Nath, and S. Pal, Phys. Rev. C 91, 034619 (2015).

[14] Y. Le Beyec, M. Lefort, and M. Sarda, Nucl. Phys. A 192, 405 (1972).

[15] D. Vermeulen, H. G. Clerc, and C. C. Sahm, Z. Phys. A 318, 157 (1984).

[16] D. Ackermann, Eur. Phys. J. A 20, 151 (2004).

[17] A. N. Andreyev, D. Ackermann, S. Antalic, I. G. Darby, S. Franchoo, F. P. Heßberger, S. Hofmann, M. Huyse, P. Kuusiniemi, B. Lommel, B. Kindler, R. Mann, G. Münzenberg, R. D. Page, S. Saro, B. Sulignano, B. Streicher, K. Van de Vel, P. Van Duppen, and D. R. Wiseman, Phys. Rev. C 72, 014612 (2005).
[18] J. Gehlot, S. Nath, Tathagata Banerjee, Ish Mukul, R. Dubey, A. Shamlath, P. V. Laveen, M. Shareef, Md. M. Shaikh, A. Jhingan, N. Madhavan, T. Rajbongshi, P. Jisha, and Santanu Pal, Proc. DAE-BRNS Symp. Nucl. Phys. 61, 404 (2016).

[19] A. Shrivastava, S. Kailas, A. Chatterjee, A. M. Samant, A. Navin, P. Singh, and B. S. Tomar, Phys. Rev. Lett. 82, 699 (1999).

[20] K. Mahata, S. Kailas, A. Shrivastava, A. Chatterjee, P. Singh, S. Santra, and B. S. Tomar, Phys. Rev. C 65, 034613 (2002).

[21] A. Chaudhuri, T. K. Ghosh, K. Banerjee, S. Bhattacharya, J. Sadhukhan, S. Kundu, C. Bhattacharya, J. K. Meena, G. Mukherjee, A. K. Saha, M. A. Asgar, A. Dey, S. Manna, R. Pandey, T. K. Rana, P. Roy, T. Roy, V. Srivastava, P. Bhattacharya, D. C. Biswas, B. N. Joshi, K. Mahata, A. Shrivastava, R. P. Vind, S. Pal, B. R. Behera, and V. Singh, Phys. Rev. C 92, 041601(R) (2015).

[22] M. Morjean, D. J. Hinde, C. Simenel, D. Y. Jeung, M. Airiau, K. J. Cook, M. Dasgupta, A. Drouart, D. Jacquet, S. Kalkal, C. S. Palshetkar, E. Prasad, D. Rafferty, E. C. Simpson, L. Tassan-Got, K. Vo-Phuoc, and E. Williams, Phys. Rev. Lett. 119, 222502 (2017).

[23] A. N. Andreyev, M. Huyse, P. Van Duppen, C. Qi, R. J. Liotta, S. Antalic, D. Ackermann, S. Franchoo, F. P. Heßberger, S. Hofmann, I. Kojouharov, B. Kindler, P. Kuusiniemi, S. R. Lesher, B. Lommel, R. Mann, K. Nishio, R. D. Page, B. Streicher, Š. Šáro, B. Sulignano, D. Wiseman, and R. A. Wyss, Phys. Rev. Lett. 110, 242502 (2013).

[24] G. K. Mehta and A. P. Patro, Nucl. Instrum. Methods A 268, 334 (1988).

[25] D. Kanjilal, S. Chopra, M. M. Narayanan, Indira S. Iyer, Vandana Jha, R. Joshi, and S. K. Datta, Nucl. Instrum. Methods A 328, 97 (1993).

[26] J. Gehlot, S. R. Abhilash, S. Ojha, D. Mehta, D. Kabiraj, and A. M. Vinodkumar, J. Radioanal. Nucl. Chem. 305, 755 (2015).

[27] N. Madhavan, S. Nath, T. Varughese, J. Gehlot, A. Jhingan, P. Sugathan, A. K. Sinha, R. Singh, K. M. Varier, M. C. Radhakrishna, E. Prasad, S. Kalkal, G. Mohanto, J. J. Das, Rakesh Kumar, R. P. Singh, S. Muralithar, R. K. Bhowmik, A. Roy, Rajesh Kumar, S. K. Suman, A. Mandal, T. S. Datta, J. Chakko, A. Choudhury, U. G. Naik, A. J. Malayadri, M. Archunan, J. Zacharias, S. Rao, Mukesh Kumar, P. Barua, 
E. T. Subramanian, K. Rani, B. P. Ajith Kumar, and K. S. Golda, Pramana J. Phys. 75, 317 (2010).

[28] A. Jhingan, Pramana J. Phys. 85, 483 (2015).

[29] S. Nath, A Monte Carlo code to model ion transport in dilute gas medium (unpublished).

[30] S. Nath, P. V. Madhusudhana Rao, S. Pal, J. Gehlot, E. Prasad, G. Mohanto, S. Kalkal, J. Sadhukhan, P. D. Shidling, K. S. Golda, A. Jhingan, N. Madhavan, S. Muralithar, and A. K. Sinha, Phys. Rev. C 81, 064601 (2010).

[31] P. D. Shidling, N. M. Badiger, S. Nath, R. Kumar, A. Jhingan, R. P. Singh, P. Sugathan, S. Muralithar, N. Madhavan, A. K. Sinha, Santanu Pal, S. Kailas, S. Verma, K. Kalita, S. Mandal, R. Singh, B. R. Behera, K. M. Varier, and M. C. Radhakrishna, Phys. Rev. C 74, 064603 (2006).

[32] E. Prasad, K. M. Varier, N. Madhavan, S. Nath, J. Gehlot, Sunil Kalkal, Jhilam Sadhukhan, G. Mohanto, P. Sugathan, A. Jhingan, B. R. S. Babu, T. Varughese, K. S. Golda, B. P. Ajith Kumar, B. Satheesh, Santanu Pal, R. Singh, A. K. Sinha, and S. Kailas, Phys. Rev. C 84, 064606 (2011).

[33] K.-T. Brinkmann, A. L. Caraley, B. J. Fineman, N. Gan, J. Velkovska, and R. L. McGrath, Phys. Rev. C 50, 309 (1994).

[34] O. B. Tarasov and D. Bazin, Nucl. Instrum. Methods B 266, 4657 (2008).

[35] A. Gavron, Phys. Rev. C 21, 230 (1980).

[36] G. Mohanto, N. Madhavan, S. Nath, J. Gehlot, Ish Mukul, A. Jhingan, T. Varughese, A. Roy, R. K. Bhowmik, I. Mazumdar, D. A. Gothe, P. B. Chavan, J. Sadhukhan, S. Pal, Maninder Kaur, Varinderjit Singh, A. K. Sinha, and V. S. Ramamurthy, Phys. Rev. C 88, 034606 (2013).

[37] V. Singh, B. R. Behera, Maninder Kaur, A. Kumar, K. P. Singh, N. Madhavan, S. Nath, J. Gehlot, G. Mohanto, A. Jhingan, I. Mukul, T. Varughese, J. Sadhukhan, S. Pal, S. Goyal, A. Saxena, S. Santra, and S. Kailas, Phys. Rev. C 89, 024609 (2014).

[38] R. Sandal, B. R. Behera, V. Singh, M. Kaur, A. Kumar, G. Kaur, P. Sharma, N. Madhavan, S. Nath, J. Gehlot, A. Jhingan, K. S. Golda, H. Singh, S. Mandal, S. Verma, E. Prasad, K. M. Varier, A. M. Vinodkumar, A. Saxena, J. Sadhukhan, and S. Pal, Phys. Rev. C 91, 044621 (2015).

[39] K. Sudarshan, R. Tripathi, S. Sodaye, S. K. Sharma, P. K. Pujari, J. Gehlot, N. Madhavan, S. Nath, G. Mohanto, I. Mukul, A. Jhingan, and I. Mazumdar, Phys. Rev. C 95, 024604 (2017).
[40] A. Shamlath, E. Prasad, N. Madhavan, P. V. Laveen, J. Gehlot, A. K. Nasirov, G. Giardina, G. Mandaglio, S. Nath, Tathagata Banerjee, A. M. Vinodkumar, M. Shareef, A. Jhingan, T. Varughese, D. V. G. R. K. S. Kumar, P. Sandya Devi, Khushboo, P. Jisha, Neeraj Kumar, M. M. Hosamani, and S. Kailas, Phys. Rev. C 95, 034610 (2017).

[41] P. Sharma, B. R. Behera, Ruchi Mahajan, Meenu Thakur, Gurpreet Kaur, Kushal Kapoor, Kavita Rani, N. Madhavan, S. Nath, J. Gehlot, R. Dubey, I. Mazumdar, S. M. Patel, M. Dhibar, M. M. Hosamani, Khushboo, N. Kumar, A. Shamlath, G. Mohanto, and S. Pal, Phys. Rev. C 96, 034613 (2017).

[42] W. Reisdorf, Z. Phys. A 300, 227 (1981).

[43] W. Reisdorf and M. Schadel, Z. Phys. A 343, 47 (1992).

[44] http://www.nndc.bnl.gov/

[45] R. Bass, Lect. Notes Phys. 117, 281 (1980).

[46] A. B. Quint, W. Reisdorf, K. H. Sehmidt, P. Armbruster, F. P. Hessberger, S. Hofmann, J. Keller, G. Munzenberg, H. Stelzer, H.-G. Clerc, W. Morawek, and C. C. Sham, Z. Phys. A 346, 119 (1993).

[47] R. N. Sagaidak, in Proceedings of the International Conference on Shells-50, edited by Yu. Ts. Oganessian and R. Kalpakchieva (World Scientific, Singapore, 2000), p. 199.

[48] A. V. Ignatyuk, G. M. Smirenkin, and A. Tishin, Sov. J. Nucl. Phys. 21, 255 (1975); Yad. Fiz. 21, 485 (1975).

[49] G. Audi and A. H. Wapstra, Nucl. Phys. A 595, 409 (1995).

[50] J. P. Blocki, H. Feldmeier, and W. J. Swiatecki, Nucl. Phys. A 459, 145 (1986).

[51] Jin Gen-Ming, Xie Yuan-Xiang, Zhu Yong-Tai, Shen WenGing, Sun Xi-Jun, Guo Jun-Sheng, Liu Guo-Xing, Yu Ju-Sheng, Sun Chi-Chang, and J. D. Garrett, Nucl. Phys. A 349, 285 (1980).

[52] H. C. Britt and A. R. Quinton, Phys. Rev. 120, 1768 (1960).

[53] C. R. Morton, D. J. Hinde, J. R. Leigh, J. P. Lestone, M. Dasgupta, J. C. Mein, J. O. Newton, and H. Timmers, Phys. Rev. C 52, 243 (1995).

[54] D. J. Hinde, M. Dasgupta, and A. Mukherjee, Phys. Rev. Lett. 89, 282701 (2002).

[55] D. J. Hinde, A. C. Berriman, M. Dasgupta, J. R. Leigh, J. C. Mein, C. R. Morton, and J. O. Newton, Phys. Rev. C 60, 054602 (1999).

[56] A. Mukherjee, D. J. Hinde, M. Dasgupta, K. Hagino, J. O. Newton, and R. D. Butt, Phys. Rev. C 75, 044608 (2007). 